\title{
Inhibition of ice nucleation by slippery liquid- infused porous surfaces (SLIPS)
}

\section{Citation}

Wilson, Peter W., Weizhe Lu, Haojun Xu, Philseok Kim, Michael J. Kreder, Jack Alvarenga, and Joanna Aizenberg. 2013. Inhibition of Ice Nucleation by Slippery Liquid-Infused Porous Surfaces (SLIPS). Phys. Chem. Chem. Phys. 15, no. 2: 581-585. doi:10.1039/c2cp43586a.

\section{Published Version}

doi:10.1039/c2cp43586a

\section{Permanent link}

http://nrs.harvard.edu/urn-3:HUL.InstRepos:27663226

\section{Terms of Use}

This article was downloaded from Harvard University's DASH repository, and is made available under the terms and conditions applicable to Open Access Policy Articles, as set forth at http:// nrs.harvard.edu/urn-3:HUL.InstRepos:dash.current.terms-of-use\#OAP

\section{Share Your Story}

The Harvard community has made this article openly available.

Please share how this access benefits you. Submit a story.

\section{Accessibility}




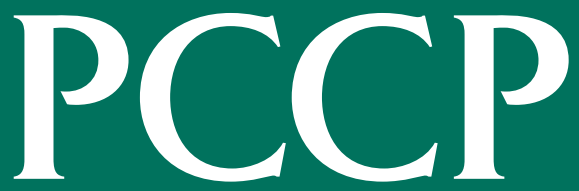

\section{Accepted Manuscript}

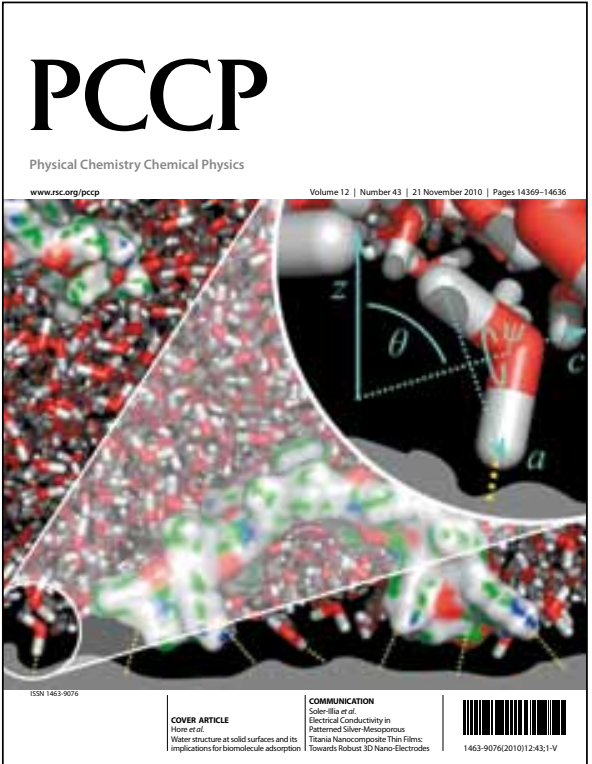

This is an Accepted Manuscript, which has been through the RSC Publishing peer review process and has been accepted for publication.

Accepted Manuscripts are published online shortly after acceptance, which is prior to technical editing, formatting and proof reading. This free service from RSC Publishing allows authors to make their results available to the community, in citable form, before publication of the edited article. This Accepted Manuscript will be replaced by the edited and formatted Advance Article as soon as this is available.

To cite this manuscript please use its permanent Digital Object Identifier $\left(\mathrm{DO}{ }^{\circledR}{ }^{\circledR}\right.$, which is identical for all formats of publication.

More information about Accepted Manuscripts can be found in the

\section{Information for Authors.}

Please note that technical editing may introduce minor changes to the text and/or graphics contained in the manuscript submitted by the author(s) which may alter content, and that the standard Terms \& Conditions and the ethical guidelines that apply to the journal are still applicable. In no event shall the RSC be held responsible for any errors or omissions in these Accepted Manuscript manuscripts or any consequences arising from the use of any information contained in them. 


\title{
Inhibition of Ice Nucleation by Slippery Liquid-Infused Porous Surfaces
} (SLIPS)

\author{
Peter W. Wilson ${ }^{1 *}$, Weizhe $\mathrm{Lu}^{1}$, Haojun $\mathrm{Xu}^{1}$, Philseok Kim ${ }^{2}$, \\ Michael J. Kreder ${ }^{3}$, Jack Alvarenga ${ }^{4}$, and Joanna Aizenberg ${ }^{2}$
}

1. School of Life and Environmental Sciences, University of Tsukuba, 1-1-1 Tennodai, Tsukuba, Japan

2. Wyss Institute for Biologically Inspired Engineering, Harvard University, Cambridge, MA 02138, USA

3. School of Engineering and Applied Sciences, Harvard University, Cambridge, MA 02138, USA

4. Department of Chemistry and Chemical Biology, Harvard University, Cambridge, MA 02138, USA

* to whom correspondence should be addressed

\begin{abstract}
Ice repellent coatings have been studied and keenly sought after for many years, where any advances in the durability of such coatings will result in huge energy savings across many fields. Progress in creating anti-ice and anti-frost surfaces has been particularly rapid since the discovery and development of slippery, liquid infused porous surfaces (SLIPS). Here we use SLIPS-coated differential scanning calorimeter (DSC) pans to investigate the effects of the surface modification on the nucleation of supercooled water. This investigation is inherently different from previous studies which looked at the adhesion of ice to SLIPS surfaces, or the formation of ice under high humidity conditions. Given the stochastic nature of nucleation of ice from supercooled water, multiple runs on the same sample are needed to determine if a given surface coating has a real and statistically significant effect on the nucleation temperature. We have cycled supercooling to freezing and then thawing of deionized water in hydrophilic (untreated aluminum), hydrophobic, superhydrophobic, and SLIPS-treated DSC pans multiple times to determine the effects of surface treatment on the nucleation and subsequent growth of ice. We find that SLIPS coatings lower the nucleation temperature of supercooled water in contact with statistical significance and show no deterioration or change in the coating performance even after 150 freeze/thaw cycles.
\end{abstract}




\section{Background}

Current state-of-the-art liquid repellent surfaces have, in some cases, been based on the leaves of the lotus plant which resist water due to the hierarchical micro/nano-textures on the surface with hydrophobic surface chemistry. ${ }^{1,2}$ In such cases, droplets of water balance on a cushion of trapped air and on the tips of the surface and bead up. ${ }^{3}$ The so-called lotus effect, however, does not work well for low surface tension organic or complex liquids as the liquids can spread along the surface texture and displace the trapped air. ${ }^{4}$ Moreover, if the surface is damaged, or subjected to extreme conditions (i.e. freezing temperatures and high humidity), liquid drops tend to pin to, or sink into, the textures rather than roll away. ${ }^{5}$ To date it has generally proven costly and difficult to manufacture surfaces based on the lotus strategy.

A large amount of work has been done to adapt these lotus leaf-based superhydrophobic surfaces to anti-ice applications, showing reduced ice adhesion ${ }^{7,8}$ and ice nucleation under certain conditions; however, these surfaces have been shown to fail under high humidity conditions and surface textures may serve to facilitate ice nucleation. ${ }^{9}$ Another, perhaps more promising, approach is one where ultrasmooth and chemically homogeneous slippery surfaces are created by infusing a nano/microstructured porous material with a lubricating fluid thus replacing the trapped air with a liquid that is immiscible with the fluid we want to repel. Such surfaces have been named Slippery Liquid-Infused Porous Surfaces, or SLIPS. ${ }^{9,10}$ Some of the recent work using SLIPS has focused not only on liquid repellency but also the decreased rate of ice accretion under high humidity conditions and reduced ice adhesion. ${ }^{9}$ There are significant driving forces, for both economic and industrial efficiency, to create rugged ice-phobic surfaces. ${ }^{11-16}$ The comparison between water wettability and ice adhesion is a somewhat different problem than the effects of SLIPS on ice nucleation with a supercooled solution in contact with the coating.

The concept of a SLIPS layer is shown in Figure 1 which simply demonstrates the trapping of a lubricating liquid within a nano-structured, functionalized matrix. Water then sits atop the matrix and the immiscibility assures non-adherence to the surface. The molecularly smooth and chemically homogeneous lubricating liquid layer provides extremely low sliding angle and contact angle hysteresis, typically less than $2^{\circ}$. The question of whether ice nucleation will be affected in any way by such a layer is addressed below. 


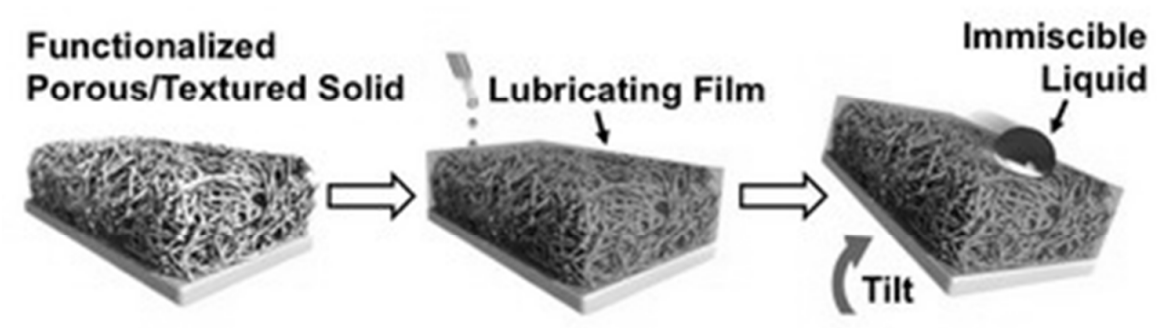

Fig. 1. Schematic showing the principle of the Slippery Liquid-Infused Porous Surface (SLIPS). (image courtesy of Peter Allen and James C. Weaver)

There have been previous studies on the dynamics of ice nucleation on water repellent surfaces, but those studies typically measured the time a sample spent supercooled, known as the lag time, or induction time. ${ }^{17}$ Unfortunately, lag times can vary by orders of magnitude for the same sample, in the same container, even when the liquid is supercooled to a given constant temperature. ${ }^{18}$ Then the measurement must be repeated many (hundreds of) times to accurately determine the effect of any change in the container, such as an additional surface coating layer. The protocol used in the current investigation is to cool a solution below its melting point and to keep cooling until it freezes, and then to warm the sample to melt the ice and repeat that process many times to determine the average nucleation temperature for that solution, in that container.

This study aims to determine if the nucleation temperature of water in a given container is lowered by the addition of a SLIPS layer and to initially quantify any such change. We are not seeking to investigate ice nucleation of a bouncing droplet, or ice adhesion properties, but rather simple heterogeneous nucleation of supercooled water in contact with a SLIPS coating.

\section{Materials and Methods}

Previous work has investigated heterogeneous nucleation of ice from water using a technique known as an automatic lag time apparatus (ALTA), which relied on the liquid sample being placed in a small test tube and light being transmitted through the liquid or scattered following subsequent freezing of the sample. ${ }^{19-21}$ We have yet to find a way to treat the insides of the glass tubes uniformly with a SLIPS layer and so it was determined that aluminum DSC pans would be suitable containers in these measurements. There are reports in the literature of the heterogeneous freezing of water in DSC pans, since water is often used as a calibrating agent, or control. 
Previous works report that the typical sample volume (5 or $10 \mu \mathrm{L})$ of water will supercool and freeze in DSC pans at temperatures ranging between about $-21^{\circ} \mathrm{C}$ and $-25^{\circ} \mathrm{C}^{22}$

Aluminum DSC pans were purchased from TA Instruments (part no. T-111121) and were treated in several steps, labeled here 0 through 8 :

0 . Untreated in any way and used as supplied.

1. Ultrasonic rinse in acetone, 15 minutes and then dried with nitrogen.

2. Placed the DSC pan inside a holder to minimize deformation during sand blasting, and sand blasted (150 grit alumina, at $80 \mathrm{psi}$ ) for 10 seconds.

3. Ultrasonic rinse in acetone, 15 minutes and dried with nitrogen.

4. Boehmite $(\gamma-\mathrm{AlO}(\mathrm{OH}))$ formation by placing the DSC pan in boiling deionized water (DIW) for 10 minutes.

0 . Rinse with DIW.

1. Fluoroalkyl surface modification in $1 \mathrm{wt}$. \% fluoroalkyl phosphate surfactant

(FS-100, Mason Chemical Co.) solution in 95:5 (v/v) ethanol: water at $70^{\circ} \mathrm{C}, 1$ hour.

7. Rinse with ethanol.

8. Add Krytox GPL 101 (DuPont Performance Lubricants, Wilmington, DE 19880-0702, USA) in small quantity (estimated at 0.3 to $0.4 \mu \mathrm{L}$ ) to form a SLIPS layer within the nanostructures.

We used four types of treatment for our investigations:

1. Untreated pans (labeled N) were used as supplied by the manufacturer.

2. The hydrophobic samples (labeled T) had only Boehmite (aluminum oxy hydroxide) and modified with long chain alkyl groups (step 1 then steps 5-7 with octadecylphosphonic acid in place of fluoroalkyl phosphate surfactant). These pans had only nanostructures from Boehmite formation, but were not superhydrophobic and had no microscale structure.

3. The superhydrophobic samples (labeled $\mathrm{H}$ ) had gone through all the steps 1-7 and had hierarchical structures, i.e. microscale texture from the sandblasting and nanoscale texture on top of it from Boehmite formation. That is, the surface was covered with a molecular layer of fluorinated hydrocarbon and a $\sim 300 \mathrm{~nm}$ thick layer of Boehmite.

4. The SLIPS coated pans (labeled S) had all 8 steps performed on them. 
Figure 2 shows a schematic of the setup for cooling and heating of the sample pans. A Pt100 temperature sensor was placed on top of the thermal transfer material, close to, but not inside, the DSC pan to avoid the sensor being a site for nucleation. In all cases, $10 \mu \mathrm{L}$ of deionized water was used (from the same bulk sample). The room temperature was $20{ }^{\circ} \mathrm{C}$ and although the humidity outside the pan varied, the cover slip tended to seal sufficiently well that inside the pan was at a relatively constant vapor pressure.

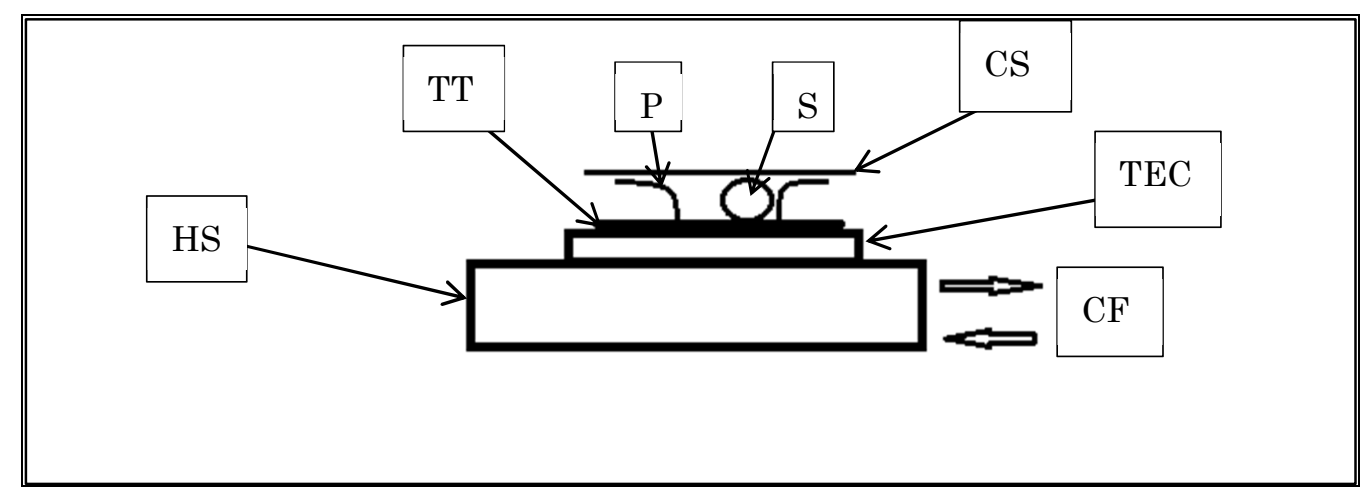

Fig. 2. Schematic of the experimental arrangement showing the heat sink (HS), thermoelectric cooling (TEC), cooling fluid (CF), thermal transfer material (TT), DSC pan (P) with liquid sample (S) inside and cover slip (CS). Freezing was detected optically from above. The pan and sensor were insulated (not shown) from the laboratory air to minimize the thermal lag between the sensor and the liquid sample.

Following protocol from previous ALTA ${ }^{19,20}$ work, we repeatedly cooled the pan and sample, observed freezing and then reversed the current to the thermo-electric cooling (Peltier) unit, heating and thawing the sample. A schematic of the cooling and warming cycle is shown in Figure 3. Each run was performed without changing the sample solution and we operated in close to linear cooling modes at rates $\alpha(\mathrm{i})$. Cooling from warm was rapid at $\alpha_{1}=20{ }^{\circ} \mathrm{C} \mathrm{min}^{-1}$ until $0{ }^{\circ} \mathrm{C}$ and thereafter $\alpha_{2}=5{ }^{\circ} \mathrm{C} \mathrm{min}^{-1}$ until freezing occurred. Warming was also at $\alpha_{3}=20^{\circ} \mathrm{C}$ min-1 and the sample was held at $30^{\circ} \mathrm{C}$ for a few seconds to ensure complete melting of any ice before beginning the next freeze cycle. 


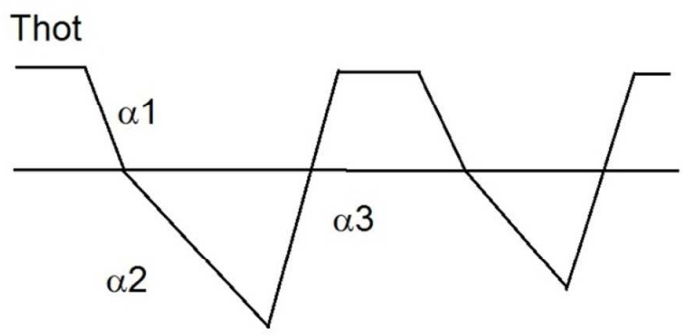

Figure 3. Schematic of the cooling and warming rates for each of the 150 cycles for one run series on a given sample.

Note also that what we measured is the temperature at which macroscopic freezing is observed, not the nucleation event per se. However, since the sample is small and supercooled to typically $-24{ }^{\circ} \mathrm{C}$, the freezing process is so rapid that the lag between nucleation and obvious sample freezing is insignificant. The data collected are simply the temperature at which a given sample froze in each of 150 runs on the same sample, in the same pan.

\section{Results}

Plots from ALTA-type measurements are known as Manhattans, ${ }^{18-21}$ and give the temperature of each freezing event extended over many test cycles. A typical data set is shown in Figure 4 and the stochastic nature of nucleation is evident, where the freezing temperature of one run is independent of previous or future runs. If the stochastic nature lasts for the 150 cycles and there is no obvious slope or steps in the plot, then we can assume that the sample does not change during those freeze/thaw cycles. 


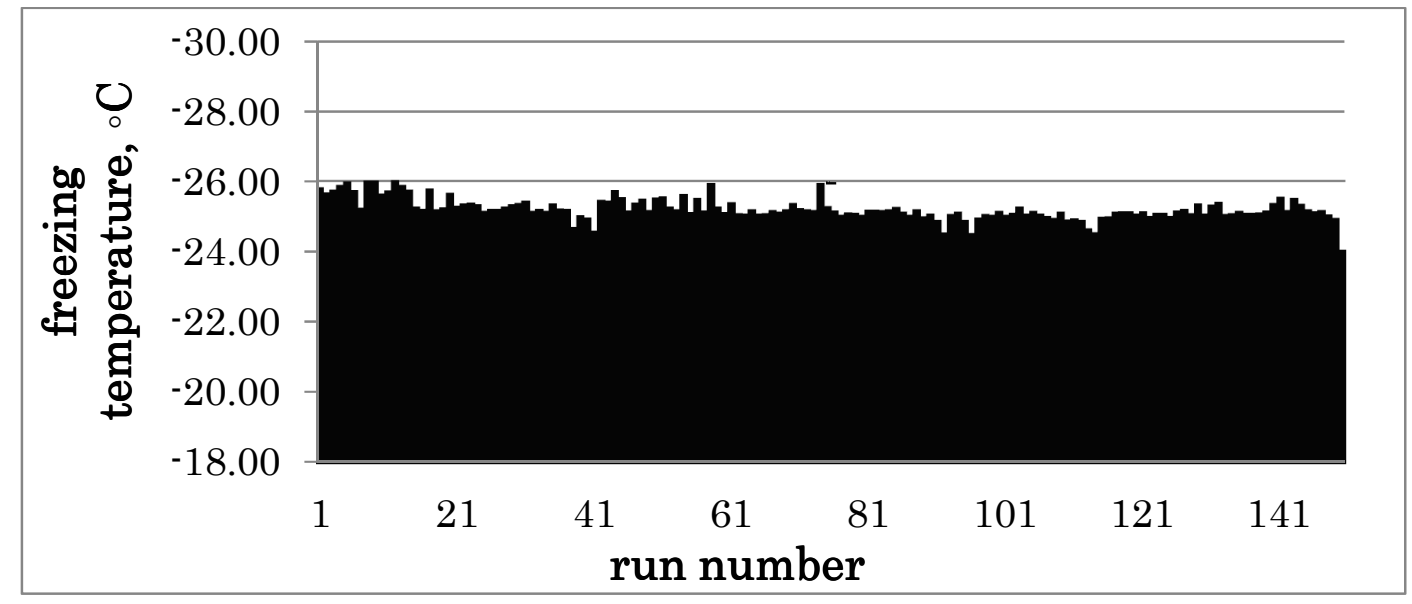

Fig. 4. A typical data set from an ALTA setup is shown here (in this case for an untreated, N, pan) and the stochastic nature of nucleation is evident, as each run freezes at a different temperature. This type of plot has been called a Manhattan, by Haymet and co-workers. $^{18-21}$

When data from ALTA, as shown in Figure 4, is analyzed further it produces a survival curve, such as that in Figure 5. The natural definition of the nucleation temperature is the temperature at which the survival curve crosses the $50 \%$ unfrozen mark, namely the temperature at which, on average, the sample has frozen half of the times. We call this temperature the T50. For the data shown in Figure 5 the proposed T50 is $-24.9{ }^{\circ} \mathrm{C}$. However, this survival curve also provides experimental error bars for the nucleation temperature of that sample. By measuring the 10-90 width (the range of temperature over between where the sample is unfrozen $90 \%$ of the time to the temperature where the sample is unfrozen $10 \%$ of the time), upper and lower bounds emerge naturally from this analysis. For the data shown in Figure 5, the 10-90 width is $1.9{ }^{\circ} \mathrm{C}$. This spread in the temperature of nucleation for the exact same sample demonstrates further the necessity of collecting many repetitions. 


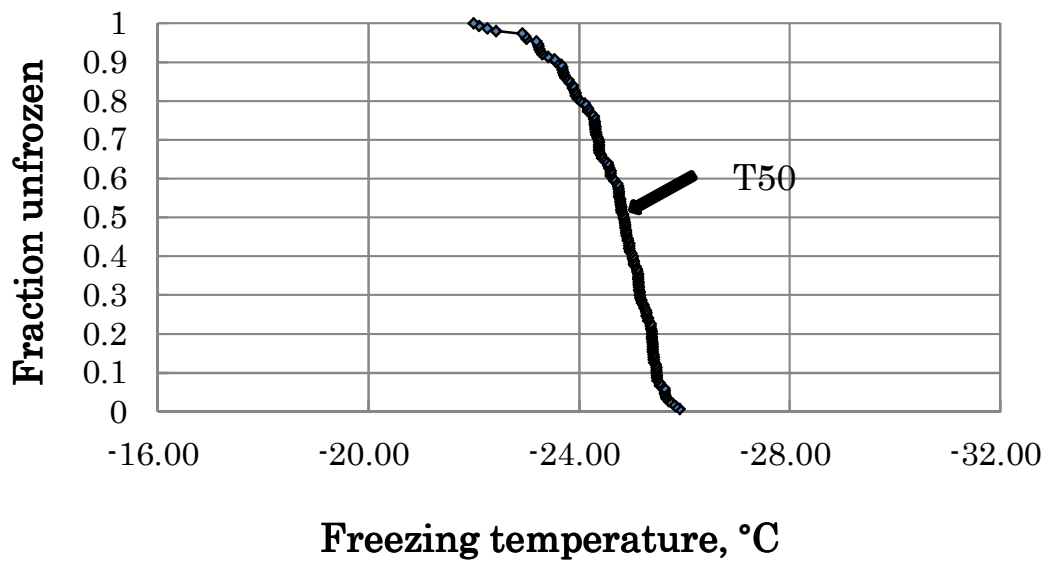

Fig. 5. This plot shows a typical survival curve, and is data from a SLIPS coated, S, pan. When data such as that shown in Figure 4 are re-plotted, they produce a survival, or probability distribution, curve such as the one shown here. The temperature at which the sample has frozen half of the 150 times is called the "T50" and represents the average nucleation temperature of that sample, in that container.

If, for example, two survival curves have similar slopes, and thus similar 10-90 widths, and are offset by 1 or $2{ }^{\circ} \mathrm{C}$ then we are certain that they have different nucleation temperatures and that the stochastic nature of nucleation is not simply blurring the picture. With these complete measurements, we then have the ability to see if changes to the sample container, such as coatings, affect the T50 value. Of course, changing pans can, and will, always change the T50. In an unchanging and clean liquid sample it is usually a defect, or some microstructure on the container wall, which is the best, or most efficient, nucleation site which initiates the nucleation and freezing process. If, however, the liquid contains a good nucleator such as a silver iodide (AgI) crystal, a piece of insoluble dirt or sand, or even certain bacteria, then that may be the best nucleation site. In which case nucleation will occur at a higher temperature than it would have without the object.

Results from our measurements with the four types of pan treatment are shown in Figure 6. In summary, we find that hydrophobized pans (T) seem to have a slightly warmer nucleation temperature than untreated, hydrophilic $(\mathrm{N})$ pans - but with two of each sample, there is a chance that any difference is due simply to inter-sample variability and is independent of the surface treatment. 
Perhaps more importantly we also see that superhydrophobic pans $(\mathrm{H})$ have a larger spread of T50s than we might have expected, and this could be explained as the heterogeneity of the nano- and microstructured surface of the aluminum serving as good nucleation sites. Firm conclusions cannot be drawn from the T50s of the lotus-leaf inspired ( $\mathrm{T}$ and $\mathrm{H}$ ) hydrophobic, or superhydrophobic surfaced, pans due to these uncertainties. It is important to note however that the spread of $\mathrm{H}$ pan T50s is larger than the 10-90 widths and this shows that the spread is not a function of the stochastic nature of nucleation but due to the difference in efficiency of the best nucleation site in one pan compare to another pan.

What we do find is that using the same superhydrophobic, $\mathrm{H}$ pans and then adding the lubricant to complete the SLIPS coating removes this possible source of error or uncertainty due to inter-sample variability. The SLIPS layer significantly changes the T50 and so produces what could be called an ice-phobic layer. Note that $\mathrm{S} 1$ is the actual H4 pan with lubricant added to turn it into a SLIPS-coated pan, similarly S2 is the actual H5 pan.

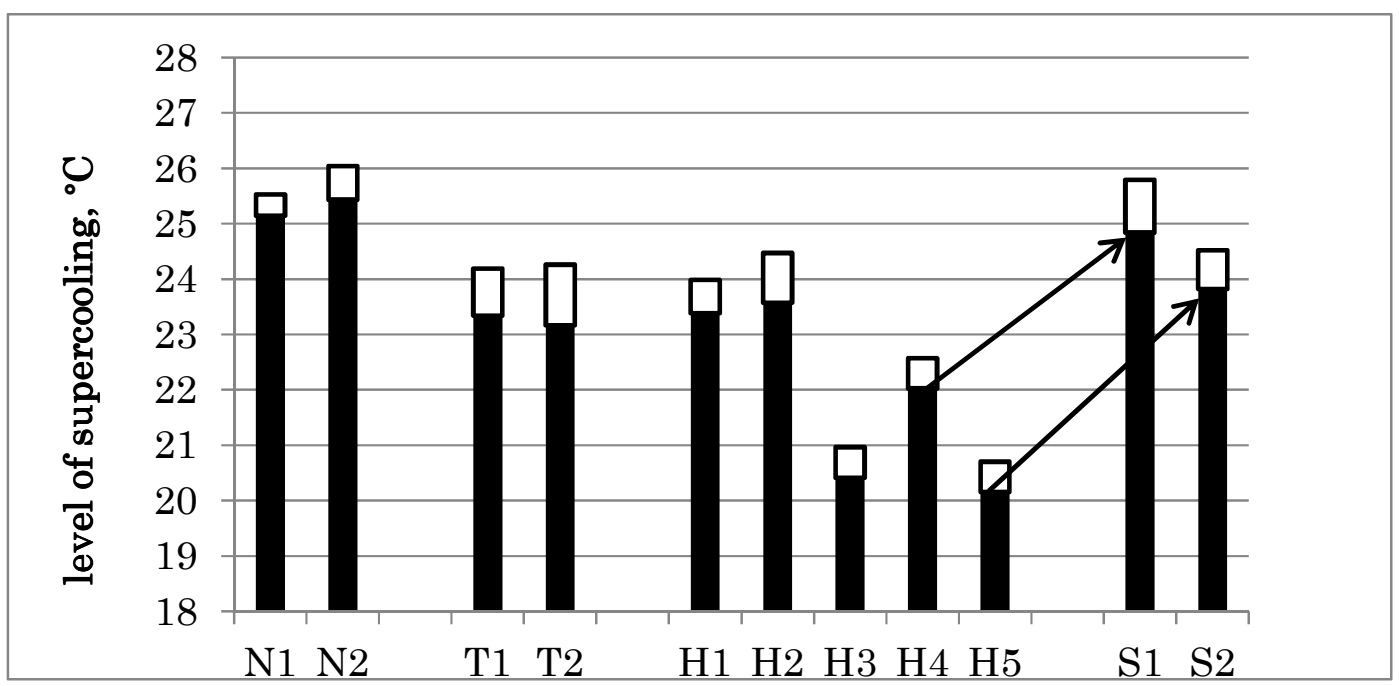

Fig. 6. A plot comparing the level of supercooling (T50s, black bars) for each type of surface treated aluminum DSC pans. Those labeled $\mathrm{N}$ are untreated, hydrophilic pans; $\mathrm{T}$ are textured, hydrophobic; $\mathrm{H}$ are superhydrophobic and $\mathrm{S}$ are SLIPS-coated pans. Note that $\mathrm{S} 1$ is the actual $\mathrm{H} 4$ pan with lubricant added to turn it into a SLIPS-coated pan, similarly S2 is the actual H5 pan. Also shown are the 10-90 widths, halved, to give an natural error bar to each T50 and survival curve. The relatively narrow 10-90 widths indicate that none of the samples changed in any measurable way during the 150 freeze thaw cycles of each of these data sets. 
Another factor to consider is that the survival curves for the SLIPS coated pans retained their shape and comparable 10-90 width. From this information, we can conclude that the surface and the ice-phobic nature of the coating did not deteriorate during the 150 freeze-thaw cycles. The 10-90 widths of all pans tested are also shown in Figure 6, where their values have been halved to show a natural error bar for each survival curve. The relatively narrow 10-90 widths indicate that there were no effects due to the freeze/thaw cycles in any of the treatments.

\section{Discussion and Conclusion}

The decrease of the level of supercooling for textured hydrophobic surfaces (T) compared with untreated $(\mathrm{N})$ pans is possibly explained by the higher density of heterogeneous nucleation sites. Since the surface of $\mathrm{T}$ pans is covered with a molecular layer of long chain hydrocarbon and a $\sim 300 \mathrm{~nm}$ thick Boehmite, it is worth considering the effects of reduced thermal conductivity arising from the oxide layer. However, in a linear cooling mode any decrease in thermal conductivity would delay the onset of nucleation and shift the T50 to colder temperatures, and this was not observed.

It may also be expected that superhydrophobic $(\mathrm{H})$ pans would increase the level of supercooling because of reduced contact area of water droplet with the aluminum pan if the droplet maintains a Cassie state, thus reducing thermal contact. However, this may only happen for a finite number of initial cycles and the repeated freezing/thawing may lead to irreversible Cassie to Wenzel transition at some point. ${ }^{23}$ If that happens, the $\mathrm{H}$ pan would effectively become a $\mathrm{T}$ pan with increased surface area in contact with the water droplet. Any such transition should make the statistics (10-90 width) of $\mathrm{H}$ broader than $\mathrm{T}$. The five $\mathrm{H}$ samples used in this investigation were made in a same batch so they should have same thickness of surface treatment. We do not see any significant broadening of the 10-90 widths for the $\mathrm{H}$ pans and so the transition, if it happened must have occurred in the first few thermal cycles. What we do notice is the spread of T50 for the $\mathrm{H}$ pans. Even though five $\mathrm{H}$ pans were treated identically they showed significantly different T50s. Since their 10-90 widths are similar we conclude that the five pans had quite different best nucleation sites, all of which were more efficient than those encountered on the untreated pan and given that they exhibit superhydrophobic behavior, this is an unusual finding which requires further study.

The SLIPS treatment provides an ultrasmooth and chemically homogeneous interface and can presumably eliminate possible nucleation sites, both at the bottom and 
the sidewall of a DSC pan. Thus, an increase of the level of supercooling is expected and this is what we found. As shown in Figure 6, S1 is the actual H4 pan with lubricant added to turn it into a SLIPS-coated pan, similarly S2 is the actual H5 pan. The change in T50s of between 3 and $4{ }^{\circ} \mathrm{C}$ is significant and equally significant is the lack of broadening of the 10-90 widths during 150 freeze thaw cycles. Previous work using ALTA and a hydrophobic self-assembled monolayer-coated container showed that the T50 could be shifted about $1.2{ }^{\circ} \mathrm{C}$ to colder temperatures. ${ }^{24}$ Thus, the findings here with a SLIPS-coated container are indeed significant and certainly warrant further investigation.

The SLIPS coated aluminum pans show significantly increased supercooling ability and show no detrimental effects after 150 freeze/thaw cycles. Additional improvements in uniformity of surface roughness on the treated surfaces are expected to reduce the number of nucleation sites that may protrude from the SLIPS coating, and result in an enhanced supercooling effect.

Acknowledgements. We thank Dr Tony Haymet for many helpful discussions and suggestions. 


\section{References}

1. Patankar, N. A. Mimicking the Lotus Effect: Influence of Double Roughness Structures and Slender Pillars. Langmuir, 2004, 20, 8209- 8213.

2. Yao, X., Song, Y. and L. Jiang. Applications of Bio-Inspired Special Wettable Surfaces. Advanced Materials, 2011, 23, 719.

3. Jung, Y. C., and B. Bhushan. Dynamic Effects of Bouncing Water Droplets on Superhydrophobic Surfaces. Langmuir, 2008, 24, 6262-6269.

4. Tuteja, A., Choi, W., Ma, M. L., Mabry, J. M., Mazzella, S. A., Rutledge, G. C., McKinley, G. H. and R. E. Cohen. Designing Superoleophobic Surfaces. Science, 2007, 318, 1618-1622.

5. Kulinich, S. A. and M. Farzaneh. How Wetting Hysteresis Influences Ice Adhesion Strength on Superhydrophobic Surfaces. Langmuir, 2009, 25, 8854-8856.

6. Sarkar, D. K. and M. Farzaneh Superhydrophobic Coatings with Reduced Ice Adhesion. J. Adhes. Sci. Technol., 2009, 23, 1215- 1237.

7. Cao, L. L., Jones, A. K., Sikka, V. K., Wu, J. Z. and D. Gao. Anti-icing Superhydrophobic Coatings. Langmuir, 2009, 25, 12444- 12448.

8. Mishchenko, L., Hatton, B., Bahadur, V., Taylor, J. A., Krupenkin, T. and J. Aizenberg. Design of Ice-Free Nanostructured Surfaces Based on Repulsion of Impacting Water Droplets. ACS Nano, 2010, 4, 7699-7707.

9. Jung, S.; Dorrestijn, M., Raps, D., Das, A., Megaridis, C. M. and D. Poulikakos. Are Superhydrophobic Surfaces Best for Icephobicity? Langmuir 2011, 27, 30593066

10. Wong, T.-S., Kang, S. H., Tang, S. K. Y., Smythe, E. J., Hatton, B. D., Grinthal, A. and J. Aizenberg. Bioinspired Self-Repairing Slippery Surfaces with Pressure-Stable Omniphobicity. Nature, 2011, 477, 443-447.

11. Kim, P., Wong, T. S., Alvarenga, J., Kreder, M. J., Adorno-Martinez, W. E. and J. Aizenberg. Liquid-Infused Nanostructured Surfaces with Extreme Anti-Ice and Anti-Frost Performance. ACS Nano, June 10, 2012, DOI: 10.1021/nn302310q

12. Tourkine, P., Le Merrer, M. and D. Quere. Delayed Freezing on Water Repellent Materials. Langmuir, 2009, 25, 7214- 7216.

13. He, M., Wang, J. X., Li, H. L., Jin, X. L., Wang, J. J., Liu, B. Q. and Y. L. Song. Super-Hydrophobic Film Retards Frost Formation. Soft Matter, 2010, 6, 2396- 
2398.

14. Meuler, A. J., McKinley, G. H. and R. E. Cohen. Exploiting Topographical Texture to Impart Icephobicity. ACS Nano, 2010, 4, 7048-7052.

15. Meuler, A. J., Smith, J. D., Varanasi, K. K., Mabry, J. M., McKinley, G. H. and R. E. Cohen. Relationships Between Water Wettability and Ice Adhesion. ACS Applied Materials and Interfaces, 2010, 2, 3100-3110.

16. Bahadur, V., Mishchenko, L., Hatton, B., Taylor, J. A., Aizenberg, J. and T. Krupenkin. Predictive Model for Ice Formation on Superhydrophobic Surfaces. Langmuir, 2011, 27, 14143-14150.

17. Alizadeh, A., Yamada, M., Li, R., Shang, W., Otta, S., Zhong, S., Ge, L., Dhinojwala, A., Conway, K. R., Bahadur, V., Vinciquerra, A. J., Stephens, B. and M. L. Blohm. Dynamics of Ice Nucleation on Water Repellent Surfaces. Langmuir, 2012, 28 (6), pp 3180-3186.

18. Barlow, T. W. and A. D. J. Haymet. ALTA: An automated lag-time apparatus for studying nucleation of supercooled liquids. Rev. Sci. Instrum. 1995, 66 (4) 2996-3007.

19. Wilson, P. W., Heneghan, A. F. and A. D. J. Haymet. Ice nucleation in Nature: supercooling point measurement and the role of heterogeneous nucleation. Cryobiology, 2003, 46, 88-98.

20. Heneghan, A. F., Wilson, P. W. and A. D. J. Haymet. Statistics of heterogeneous nucleation of supercooled water, and the effect of an added catalyst. Proc. Natl. Acad. Sci. 2002, 99. 9631-9634.

21. Henaghan, A., P. W. Wilson, G. Wang and A. D. J. Haymet. Liquid-to-Crystal Nucleation: Automated Lag-Time Apparatus to study supercooled liquids. J. Chem. Phys. 2001, 115, 7599.

22. Wilson, P. W., J. W. Arthur and A. D. J. Haymet. Ice premelting during differential scanning calorimetry. Biophysical. J. 1999, 77, 2850-2855.

23. Bahadur, V. and S. V. Garimella. Preventing the Cassie-Wenzel Transition Using Surfaces with Non-communicating Roughness Elements. Langmuir. 2009, 25, 4815-4820.

24. Heneghan, A. F., H. J. Moore, T. R. Lee and A. D. J. Haymet. Statistics of 
heterogeneous nucleation of supercooled aqueous solutions in a self-assembled monolayer-coated container. Chem. Phys. Let. 2004, 385, 441-445. 


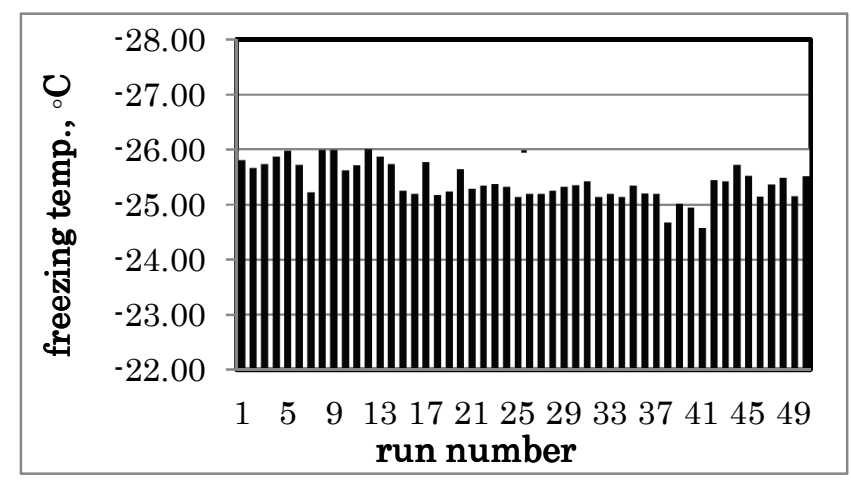

The stochastic nature of ice nucleation can hide the efficiency of coatings, but SLIPS coatings do retard ice nucleation. 